\title{
Hydrodynamic Forces of a Semi-Submerged Cylinder in an Oscillatory Flow
}

\author{
Haojie Ren ${ }^{1,2}$, Shixiao Fu ${ }^{1,2, *}$, Chang Liu ${ }^{3}{ }^{-}$, Mengmeng Zhang ${ }^{1,2}$, Yuwang $\mathrm{Xu}^{1,2}$ \\ and Shi Deng 4 \\ 1 State Key Laboratory of Ocean Engineering, Shanghai Jiao Tong University, Shanghai 200240, China; \\ rhaojie@163.com (H.R.); claire_zhang@sjtu.edu.cn (M.Z.); xuyuwang@sjtu.edu.cn (Y.X.) \\ 2 Collaborative Innovation Center for Advanced Ship and Deep-Sea Exploration, Shanghai 200240, China \\ 3 Department of Mechanical Engineering, Johns Hopkins University, Baltimore, MD 21218, USA; \\ changliu@jhu.edu \\ 4 Department of Marine Technology, Norwegian University of Science and Technology, \\ 7052 Trondheim, Norway; shi.deng@ntnu.no \\ * Correspondence: shixiao.fu@sjtu.edu.cn
}

Received: 26 August 2020; Accepted: 10 September 2020; Published: 14 September 2020

Featured Application: The present work highlights a novel empirical formula to estimate both mean and fluctuating lift force on a semi-submerged cylinder under oscillatory flow. It greatly avoids the complicated and time-consuming hydrodynamic calculation problem and will be helpful to calculate the hydrodynamic force in the design of relevant marine structures, such as floating collar of fish farming.

\begin{abstract}
This work experimentally investigated the performance of hydrodynamic forces on a semi-submerged cylinder under an oscillatory flow. To generate the equivalent oscillatory flow, the semi-submerged cylinder is forced to oscillate in several combinations of different periods and amplitudes. The mean downward lift force was observed to be significant and the fluctuating lift forces show dominant frequency is twice that of oscillatory flow and amplitude that is the same as the mean lift force. Based on this main hydrodynamic feature, a novel empirical prediction formula for the lift forces on semi-submerged cylinder under oscillatory flow is proposed where the lift forces expression is proportional to the square of oscillatory flow velocity. This novel empirical formula directly assigns the fluctuating lift force with frequency twice of oscillatory flow and the amplitude that is the same as the mean lift force. This assignment of empirical lift force formula reduces parameters required to determine a dynamic lift force but is demonstrated to well predict the fluctuating lift force. The lift coefficient can reach 1.5, which is larger than the typical value 1.2 of the drag coefficient for a fully submerged cylinder with infinite depth. Moreover, relationships among hydrodynamic coefficients, Keulegan-Carpenter (KC) number, Stokes number and Froude number are studied. With the increase of KC number, the Froude number has a more significant influence on the distribution of hydrodynamic coefficients. As Froude number is increasing, the drag coefficient shows a nonlinear decay $(K C<20)$ but a linear increase $(K C>20)$, while the added mass coefficients show a nonlinear $(K C<20)$ and a linear $(K C>20)$ increase trend. The present work can provide useful references for design of the relevant marine structures and serve as the useful guideline for future research.
\end{abstract}

Keywords: semi-submerged cylinder; oscillatory flow; hydrodynamic force; free surface; empirical prediction 


\section{Introduction}

Marine structures including oil containment booms, floating tunnel, pipelines and fish-farming float collars cannot avoid the effects of an oscillatory flow derived from the wave and relative structure motions. These marine structures can be viewed as a semi-submerged cylinder under oscillatory flow. The surface distortion and water fragmentation will result in a complicated response and hydrodynamic loads. This problem is still not fully understood and threatens the safety of the relevant marine structures. Thus, it is essential to study the hydrodynamic features of semi-submerged cylinder in an oscillatory flow for the marine structures design.

The current research of flow past a cylinder contains mainly three categories including cylinders submerged in infinite depth water, cylinders near the free surface and cylinders partially submerged under the water surface. In the oscillatory flow, many focuses are on the problem of flow past a fully immersed cylinder with infinite depth. Morison et al., (1953) [1]; Morison et al., (1950) [2] first raised a formula to calculate the in-line (IL) component of hydrodynamic force, which is now known as the Morison formula. The drag coefficient $\left(C_{D}\right)$ and added mass coefficient $\left(C_{m}\right)$ in the Morison formula were found to be respectively of value 1.7 and 0.4 through an experimental study of stationary rigid cylinders under wave actions. The values of $C_{D}$ and $C_{m}$ for a cylinder under the action of waves were further determined by Keulegan (1958) [3] through Fourier analysis and it was found that the Stokes parameters have a significant influence on these coefficients. Mercier (1973) [4] measured the force in the transverse direction, drag force and added-mass force (inertia) of the fully immersed cylinder in the oscillatory flow. The drag coefficient and added mass coefficient were witnessed to have good agreements with results of Keulegan (1958) [3]. Justesen (1989) [5] and Sarpkaya (2006) [6] experimentally studied the effects of the Re number at low Keulegan-Carpenter (KC) numbers on a rigid stationary cylinder under this oscillatory flow. Moreover, Sarpkaya (1976) [7] carried out a more comprehensive experimental studies on the forces of cylinders under oscillatory flows in sinusoidal form that was generated through an oscillatory U-shaped tube. The results were systematically reported that the hydrodynamic coefficients closely related with the Re number, Keulegan-Carpenter (KC) number and the relative roughness of the cylinder surface. Moreover, Williamson (1985) [8] and Tatsumo and Bearman (1990) [9] conducted a comprehensive flow visualization of the flow past cylinder, which provided a better understanding of the underlying fluid mechanism. Therefore, the investigation of hydrodynamic loads on the fully immersed cylinder with infinite depth in an oscillatory flow is relatively comprehensive.

Different from flow past the fully submerged cylinder with infinite depth, there are few studies focusing on cylinders near the free surface and cylinders partially submerged below the water surface in an oscillatory flow. As for close to free surface cylinders, the existing research mainly focuses on the steady flow field. Roshko (1975) [10] investigated the drag and transverse forces and discussed the relationships of the drag force and the lift force respectively with a submerged gap ratio. Bearman and Zdravkovich (1978) [11] further observed that the free surface can greatly influence the response frequency and the corresponding Strouhal number decreases quickly with the submerged gap ratio decreasing. Lei et al. (1999) [12] experimentally investigated a cylinder under steady flow and highlighted the strong effects of the boundary layer development on this lift force. Different from the experimental studies, computational fluid dynamics (CFD) provides more opportunities to perform comprehensive studies on the underlying mechanisms of complicated fluid-structure interactions (FSI) problems. Reichl et al. (1997) [13] and Sheridan et al. (1995) [14] conducted numerical simulation of cylinders near a free surface under steady flow and studied corresponding motion behavior. The jet state and metastable behavior were noted by these researchers, at the free surface under certain gap ratio and Froude (Fr) number. The configuration of a partially submerged cylinder under flow is an unavoidable and actually widely observed problem for float collars of the fish farming structures and numerous studies were conducted in the literature of the flow past float collars. For example, Triantafyllou and Dimas (1989) [15] studied the stability of a semi-submerged cylinder under flow, where results are influenced by the Fr number. At all downstream locations, convective instability 
of the wake is also observed based on the computation of potential flow theory. However, potential flow theory precludes the effect of the free surface and fluid viscosity, which cannot be ignored for a partially submerged cylinder. As a result, it is questionable whether traditional diffraction and radiation theories will provide a complete answer for this problem. Kristiansen and Faltinsen (2008) [16] and Kristiansen (2010) [17] experimentally measured the sway and heave motion of a semi-submerged circular cylinder under action of waves. Fu et al. (2013) [18] experimentally investigated hydrodynamic loads of semi-submerged cylinders that are employed in fish farming systems. It is reported that the effects of free surface are critically important for the hydrodynamic coefficients including drag coefficients and added mass coefficients in the IL direction; the direction parallel to the oscillatory flow. However, hydrodynamic forces and coefficients in the cross-flow (CF) direction have not yet been revealed and considered into models and this relationship between hydrodynamic loads and Froude number has not been studied.

Recently, Ren et al. (2019) [19] conducted experiments of the partially submerged cylinder with different submerged depths in steady flow. Surprisingly, the mean downward transverse force is witnessed to be significant, which catches more and more attentions from both academia and industry. The lift coefficient was found to be greatly affected by Froude number. However, the understanding of hydrodynamic load in the CF direction on the semi-submerged cylinder under the oscillatory flow is still blank and potential influence parameters were not studied comprehensively. Therefore, it is urgent to investigate the hydrodynamic features of the semi-submerged cylinder in an oscillatory flow, especially for the lift coefficient and providing a further understanding for relevant structural design.

In this study, model tests of semi-submerged cylinders in an oscillatory flow were carried out in a towing tank. The rigid cylinder was enforced to oscillate harmonically under numerous combinations of periods and amplitudes to generate an equivalent oscillatory flow. The KC number was designed to range from 6.3 to 37.7. The Stokes number and corresponding Froude number varied from 5434.7 to $11,363.6$ and from 0.12 to 1.55 , respectively. The hydrodynamic force in both IL and CF directions and the displacement in the IL direction were measured. We also establish the method for identifying the drag and added mass coefficients in the IL direction. Based on the observations of the lift force, a novel empirical prediction method of lift force is first proposed. The features of hydrodynamic loads, the relationships between hydrodynamic coefficients and influential parameters are further studied and discussed.

The remainder of this paper is organized as what follows. Section 2 describes the experimental set-up, test arrangement and definition of the key parameters such as Froude number, Stokes number and KC number. In Section 3, the identification of the drag and added mass coefficient are presented. Then, main results including hydrodynamic load features, the relationships among the drag, added mass, lift coefficients of the semi-submerged cylinder and influential parameters as well as a novel empirical prediction method of lift force are discussed in Section 4 . The main conclusions and future work directions are summarized in Section 5.

\section{Experimental Setup}

\subsection{Test Apparatus}

The model tests are carried out at the towing tank located at Shanghai Ship and Shipping Research Institute. The main dimension of this towing tank is $192 \mathrm{~m} \times 10 \mathrm{~m} \times 4.2 \mathrm{~m}$ (length $\times$ width $\times$ depth). The test apparatus were mounted under the towing carriage, which consists of the vertical and horizontal tracks, semi-submerged cylinder and end plate. The semi-submerged cylinder model is made of polypropylene and can be regarded as a smooth rigid cylinder. The hydrodynamic diameter $\mathrm{D}$ and length $L$ of the model are respectively $0.25 \mathrm{~m}$ and $2 \mathrm{~m}$. The vertical tracks driven by serve motors are employed for adjustment of the height of cylinder to the semi-submerged state. The horizontal track drives the semi-submerged cylinder to oscillate with various amplitudes and periods. The axonometric sketch and the actual apparatus are respectively shown in Figures 1 and 2. 


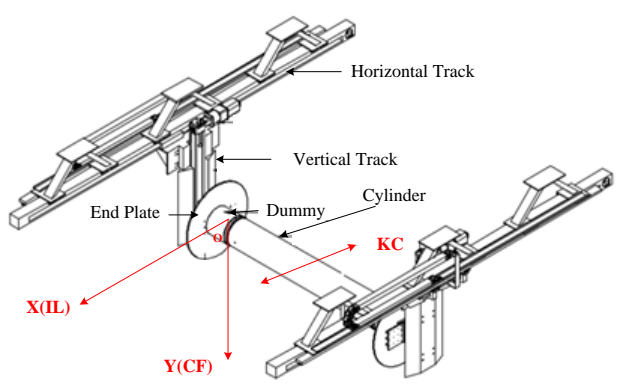

Figure 1. Sketch of the experimental apparatus for a semi-submerged cylinder under oscillatory flow; $\mathrm{X}$ and $\mathrm{Y}$ are respectively IL and CF directions.

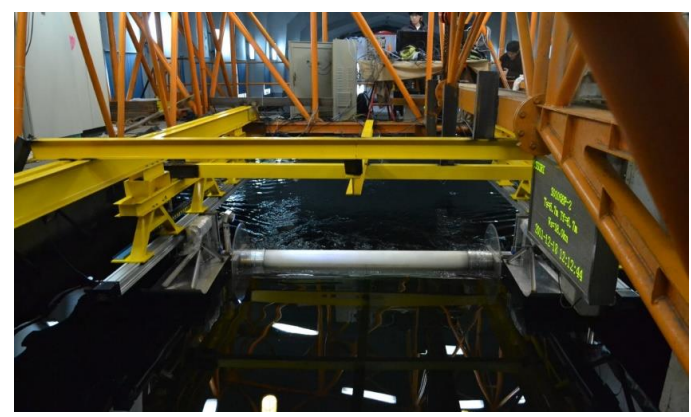

Figure 2. Photography of the experimental apparatus.

To guarantee a two-dimensional flow and avoid three-dimensional effects arising from the rigid cylinder with the finite length, we adopted two methods to avoid above problem. The first method is to assemble two dummy cylinders at both the model ends. The diameter of the dummy cylinder is the same as the rigid cylinder model. To avoid the force transducer measuring the force derived from dummy cylinders, a small gap $(<1 \mathrm{~mm})$ is left between and the rigid cylinder model and two end dummy cylinders. Another method is to assemble two circular endplates with diameter of 3 times cylinder model diameter at the end of the dummy cylinder so as to restrict the effects of other apparatus on the flow past the cylinder. The force sensors are employed to record the hydrodynamic loads on the rigid cylinder model.

To validate the measurement system and the smoothness of the cylinder, the cylinder was first fully immersed in the water and towed in steady flow. The time history of hydrodynamic force in the IL direction was recorded and shown in Figure 3. The entire time history contains four phases-initial zero, transition, stable stage and finial zero. The mean force value $F_{X M}$ in the stable stage in the IL direction is approximately $47.3 \mathrm{~N} / \mathrm{m}$ under flow velocity $\mathrm{U}$ of $0.4 \mathrm{~m} / \mathrm{s}$. The corresponding drag coefficient $C_{D}$ is calculated by,

$$
C_{D}=\frac{F_{X M}}{1 / 2 \rho D L U^{2}}
$$

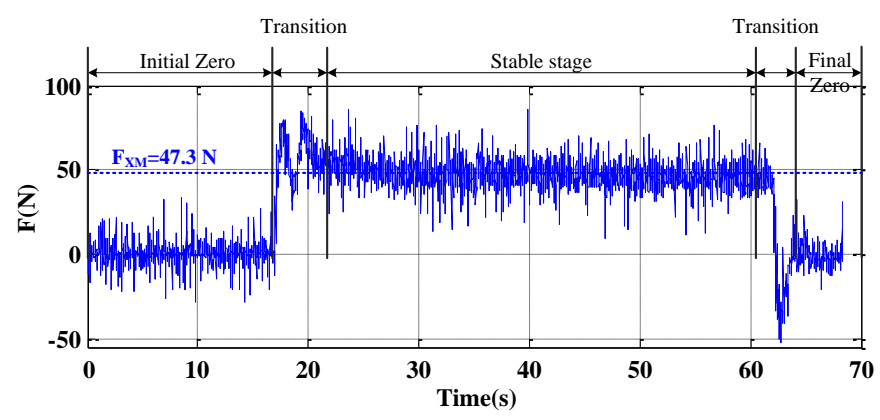

Figure 3. Time history of the hydrodynamic forces on a fully submerged cylinder under a steady flow velocity of $U=0.4 \mathrm{~m} / \mathrm{s}$. 
The $C_{D}$ is about 1.18, which is consistent with the typical value of 1.2 reported by Achenbach and Heinecke (1981) [20]. This result demonstrates our experimental apparatus is reliable.

\subsection{Test Arrangement}

To generate the equivalent horizontal oscillatory flow derived from wave action, the semi-submerged cylinder is forced to oscillate in harmonic motions in the IL direction (Chakrabarti 1987) [21]. The instantaneous displacement $X(t)$ and corresponding velocity of the forced motion $U(t)$ can be respectively indicated as what follows:

$$
\begin{gathered}
X(t)=A_{m} \sin \left(\frac{2 \pi}{T} t\right) \\
U(t)=A_{m} \frac{2 \pi}{T} \cos \left(\frac{2 \pi}{T} t\right)
\end{gathered}
$$

where $A_{m}$ and $T$ are respectively oscillation amplitude and period.

To describe the hydrodynamic features of the semi-submerged cylinder, the key parameters including KC number, Reynolds number $(\operatorname{Re})$ and Stokes number $(\beta)[3,7,18,22]$ should be considered, which can be respectively expressed as,

$$
\begin{gathered}
K C=\frac{2 \pi A_{m}}{D} \\
\operatorname{Re}=\frac{2 \pi A_{m} D}{T \cdot v} \\
\beta=\frac{\operatorname{Re}}{K C}=\frac{D^{2}}{v T}
\end{gathered}
$$

where $v=1.0 \times 10^{-6} \mathrm{~m}^{2} \mathrm{~s}^{-1}$ is the kinematic viscosity coefficient of water.

However, the free surface effects are the distinctive characteristics for flow past a semi-submerged cylinder. The Froude number $(F r)$ is also an important parameter for this hydrodynamic load on the cylinder [19] and can be defined as,

$$
F r=\frac{U_{m}}{\sqrt{g h}}
$$

where $U_{m}=A_{m} \cdot(2 \pi / T), h=0.5 \mathrm{D}, h$ is the submerged depth.

From the definition of the four parameters in Equations (4)-(7), conflicts are clear between the Froude number and Stokes number. In order to investigate the hydrodynamic force on semi- submerged cylinder in an oscillatory flow, the test cases were divided into four groups according to their KC numbers, as summarized in Table 1. Each test group maintained the same KC number with different Stokes number ranging from 5435 to 11,364 . We should note that the test cases are designed to reveal preliminary hydrodynamic force features and the cases at larger $\mathrm{KC}$ number will be a promising direction for the future work.

Table 1. Test Matrix.

\begin{tabular}{ccccccc}
\hline Case No. & $\mathbf{K C}$ & $\mathbf{A}_{\mathbf{m}}(\mathbf{m})$ & $\boldsymbol{T}(\mathbf{s})$ & $\boldsymbol{\beta}$ & $\mathbf{F r}$ & $\boldsymbol{R} \boldsymbol{e}$ \\
\hline $1-3$ & 6.3 & 0.25 & $5.5: 3: 11.5$ & $5435-11,364$ & $0.12-0.26$ & $3.4 \times 10^{4}-7.1 \times 10^{4}$ \\
$4-6$ & 18.8 & 0.75 & $5.5: 3: 11.5$ & $5435-11,364$ & $0.37-0.77$ & $1.0 \times 10^{5}-2.1 \times 10^{5}$ \\
$7-9$ & 31.4 & 1.25 & $5.5: 3: 11.5$ & $5435-11,364$ & $0.62-1.29$ & $1.7 \times 10^{5}-3.6 \times 10^{5}$ \\
$10-12$ & 37.7 & 1.5 & $5.5: 3: 11.5$ & $5435-11,364$ & $0.74-1.55$ & $2.0 \times 10^{5}-4.3 \times 10^{5}$ \\
\hline
\end{tabular}

\section{Identification Method of Hydrodynamic Coefficients}

According to the Morison equation [1,2], the hydrodynamic force in the IL direction can be decomposed into two components; that is, the drag force and the inertial force that are respectively proportional to the square of the velocity and the acceleration. The similar equation was also employed 
to describe the hydrodynamic force on floating cylinder in the horizontal direction [18]. Thus, the total force can be expressed as,

$$
F_{X P}=\frac{1}{2} C_{D} \rho D L U(t)|U(t)|+C_{m} \rho \frac{\pi D^{2}}{4} L \dot{U}(t)
$$

For convenience, it is assumed that,

$$
\begin{aligned}
& C_{1}(t)=\frac{1}{2} \rho D L U(t)|U(t)| \\
& C_{2}(t)=\rho \frac{\pi D^{2} L}{4} \dot{U}(t)
\end{aligned}
$$

Then, Equation (8) can be simplified as,

$$
F_{X P}=C_{1}(t) \cdot C_{D}+C_{2}(t) \cdot C_{m}
$$

The least squares method is used to estimate the drag coefficient $C_{D}$ and added mass coefficients $C_{m}$ in the IL direction, which minimizes the sum of the squared difference $J\left(C_{D}, C_{m}\right)$ between the predicted and measured force, which can be written as,

$$
J\left(C_{D}, C_{m}\right)=\sum_{i=1}^{n}\left[F_{X}(t)-F_{X P}(t)\right]^{2}
$$

where $F_{X}(t)$ is the measured force and $n$ is the sample number.

In order to minimize the difference between the identified and predicted values of the hydrodynamic force, Equation (11) should be satisfied,

$$
\frac{\partial J\left(C_{D}, C_{m}\right)}{\partial C_{D}}=0, \frac{\partial J\left(C_{D}, C_{m}\right)}{\partial C_{m}}=0
$$

Transforming Equation (12) into matrix form, we have

$$
\mathbf{\Lambda} \mathbf{\Lambda}^{\mathrm{T}}\left[\begin{array}{l}
C_{D} \\
C_{m}
\end{array}\right]=\mathbf{\Lambda} \mathbf{F}_{\mathbf{X}}
$$

where,

$$
\boldsymbol{\Lambda}=\left[\begin{array}{cccc}
C_{1}\left(t_{1}\right) & C_{1}\left(t_{2}\right) & \cdots & C_{1}\left(t_{n}\right) \\
C_{2}\left(t_{1}\right) & C_{2}\left(t_{2}\right) & \cdots & C_{2}\left(t_{n}\right)
\end{array}\right], \mathbf{F}_{\mathbf{X}}=\left[\begin{array}{c}
F_{X}\left(t_{1}\right) \\
F_{X}\left(t_{2}\right) \\
\vdots \\
F_{X}\left(t_{n}\right)
\end{array}\right]
$$

The drag and added mass coefficients can be expressed as,

$$
\left[\begin{array}{l}
C_{D} \\
C_{m}
\end{array}\right]=\left(\boldsymbol{\Lambda} \mathbf{\Lambda}^{\mathbf{T}}\right)^{-1} \boldsymbol{\Lambda} \mathbf{F}_{\mathbf{X}}
$$

However, there is no mature empirical equation to describe the hydrodynamic force in the CF direction. The empirical prediction of the lift force will be further studied in Section 4.3 according to the features observed by our experiments in this paper.

\section{Results and Discussion}

This section first describes the main features of hydrodynamic forces on the semi-submerged cylinder under oscillatory flow. Based on observed features, we then proposed a novel empirical 
formula that reduces the parameters required to determine lift force but is demonstrated to well predict the lift force. Furthermore, we perform a comprehensive study on the influence of KC number, Stokes number, Froude number on the drag coefficient and added mass coefficient for the IL direction as well as lift coefficients and phase difference for the prediction of lift force in the CF direction. The phenomena including slamming and overtopping are also observed and discussed.

\subsection{Hydrodynamic Forces on the Semi-Submerged Cylinder}

As shown in Figure 4, the time history of the hydrodynamic loads on a semi-submerged cylinder under the steady flow velocity of $U=0.8 \mathrm{~m} / \mathrm{s}$ was presented. The blue and red solid lines respectively represent the hydrodynamic forces in the IL and CF directions. The mean force in the IL direction $F_{X M}$ and mean lift force in the $\mathrm{CF}$ direction $F_{Y M}$ are $27.6 \mathrm{~N}$ and $178.9 \mathrm{~N}$, respectively. A novel phenomenon of an obvious mean downward lift force is witnessed for semi-submerged cylinder and reported by Ren et al. (2019) [19]. This mean lift force is greater than the drag force and should be considered in the real engineering projects.

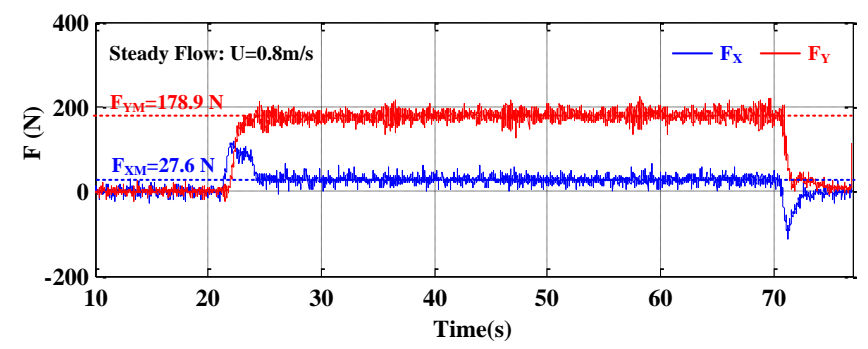

Figure 4. Time history of the hydrodynamic forces for semi-submerged cylinder under a flow velocity of $U=0.8 \mathrm{~m} / \mathrm{s}$.

For the hydrodynamic force on semi-submerged cylinder in an oscillatory flow, the time history of these forces in both IL and CF directions for KC $=18.8$ and $\beta=111,364$ is shown in Figure 5. Similar to hydrodynamic force on semi-submerged cylinder in steady flow, the mean downward force in the $\mathrm{CF}$ direction $F_{Y M}$ can be obviously witnessed. The values of $F_{Y M}$ were $162.3 \mathrm{~N}$ for $K C=18.8$ at $\beta=111,364$, while no mean values of the force in the IL direction can be observed. The mean lift force in the CF direction can reach and even exceed the fluctuating force amplitude of hydrodynamic force in the IL direction $\left(F_{X}\right)$. The fluctuating components of two forces are in the same magnitudes.

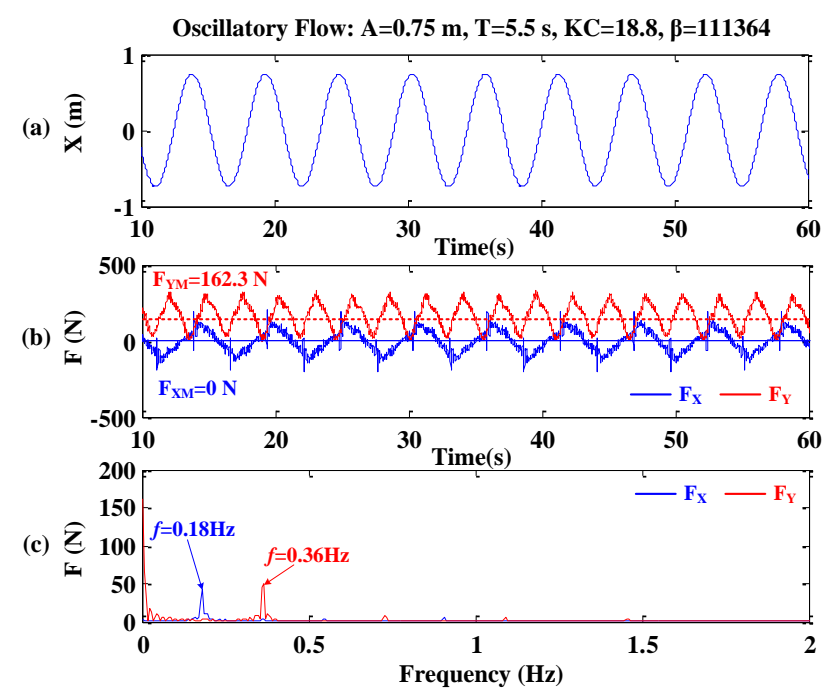

Figure 5. Time history of the hydrodynamic forces for semi-submerged cylinder under Keulegan-Carpenter $(\mathrm{KC})=18.8$ at $\beta=111,364$. (a) Time history of forced motion; (b) Time history of the hydrodynamic forces on semi-submerged cylinder; (c) The spectrum of the hydrodynamic loads. 
From the spectrum of the hydrodynamic forces in both IL and CF directions as shown in Figure 5c, the dominant frequency of force in the IL direction is the same as expected for the forced motion frequency $\left(f_{\mathrm{o}}\right)$ of $0.18 \mathrm{~Hz}$ and the response frequency in the CF direction is found to be always dominated by twice the value of $f_{\mathrm{o}}(f=0.36 \mathrm{~Hz})$ under $\mathrm{KC}=18.8$ and $\beta=111,364$. It indicates that this lift force with higher frequency could cause more severe damage to related structures and cannot be neglected.

To understand the background mechanics of the mean lift downward force in an oscillatory flow, a camera is placed on the apparatus to record the surface waveforms. Figure 6a clearly shows the recorded local surface distortions. According to the phenomenon recorded by the camera, we infer that the mean downward lift force can be attributed to the asymmetric flow field and the pressure difference between the top and bottom of the cylinder $[19,23]$ as shown in Figure 7c. Furthermore, Figure 7 presents the time history of hydrodynamic force and sketches of flow field around cylinder in one period to give more detailed features. Combining with forced motion velocity, we observed that the lift force reaches its amplitude value at around time points I, III, V. From Figure 7c, the top-sided flow velocity is more significant when the forced motion velocity reach its maximum $\left(U_{\max }\right)$. Thus, the maximum lift force value appears near the time points of $U_{\max }$.
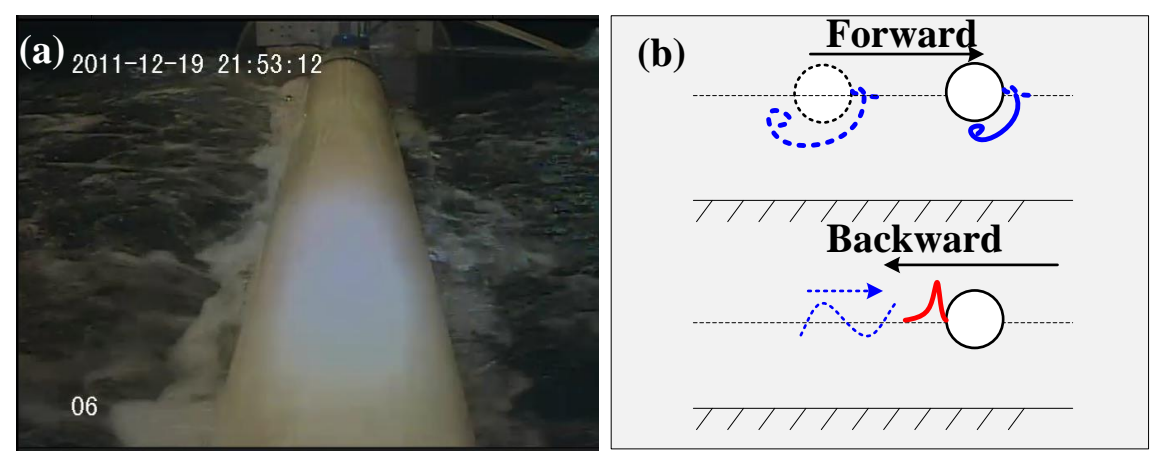

Figure 6. The pictures of slamming and its developing process sketch. (a) Pictures of slamming phenomenon; (b) Sketch of slamming developing process.
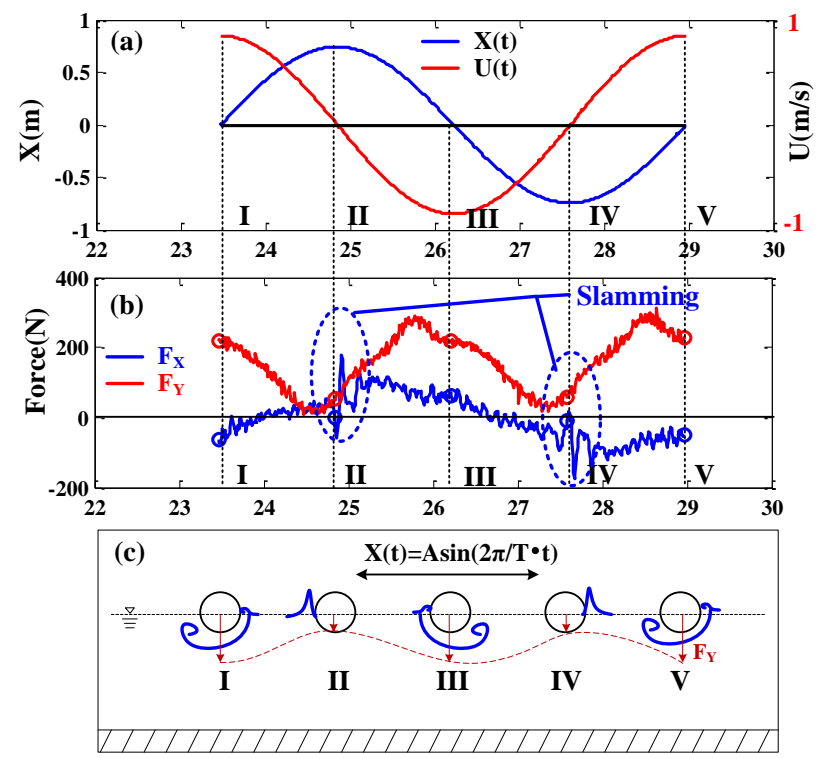

Figure 7. Time history of forced motion, hydrodynamic force and sketches of flow field around cylinder in one period under $\mathrm{KC}=18.8$ at $\beta=111,364$. (a) Time histories of forced motion displacement and velocity; (b) The hydrodynamic loads on semi-submerged cylinder; (c) Sketches of flow flied change. 
However, there is a phase difference between $F_{Y \max }$ and $U_{\max }$. These two maximum values do not reach at the same time. The maximum of force $F_{Y \max }$ is reached before the $U_{\max }$. It may be related to the effects of the exciting wave. The velocity of the exciting wave will be superimposed with the forced motion velocity. The time of the "real velocity" superimposed by the velocity of exciting wave and the forced motion reach the maximum value is not located at that of forced motion velocity amplitude. More insightful experiments and CFD simulations will be carried out in the near future to further investigate this phenomenon. This phase difference would be an important parameter to describe the relationship between the lift force and flow velocity generated by forced motion.

Beyond that, a shock signal of the force in the IL direction appears when the cylinder reverses its motion direction (II, IV). The phenomenon is caused by the "slamming" phenomenon as shown in Figure $6 \mathrm{a}$. Figure $6 \mathrm{~b}$ shows the developing process sketch of the slamming. When the cylinder move forward, the water attached on the cylinder and exciting wave will be moved in the same direction as the cylinder. As the cylinder reverse its moving direction, the attaching water and exciting wave cannot change their movement direction immediately. The cylinder will strike the exciting wave and attaching water. The water climbs significantly in an instant and results in an impulse force in the IL direction. Slamming will result in high pressure and introduce damage on local structure and subsequent failure of the entire system. Thus, it is necessary to take some considerations to the local structure designs.

\subsection{Empirical Prediction of the Lift Force for Semi-Submerged Cylinder in Oscillatory Flow}

As previously mentioned in Section 4.1, the lift force fluctuates at a frequency twice the forced motion frequency and an obvious phase difference between the force and velocity motion can be observed. Moreover, the mean value is found to be the main feature of the lift force in the CF direction. However, there is no empirical formula for lift force on semi-submerged cylinder in an oscillatory flow. In the real engineering project design, it is very difficult to efficiently determine the hydrodynamic force on these relevant marine structures. To reasonably describe the relationship between the lift force and flow velocity, above main features should be satisfied and thus we further propose the application of this empirical prediction of the lift force to partially submerged cylinder under an oscillatory flow:

$$
F_{Y P}=\frac{1}{2} \rho C_{L} D L[U(t+\tau)]^{2}=\frac{1}{2} \rho C_{L} D L U_{m}^{2}\left[\cos \left(\frac{2 \pi}{T} t+\frac{2 \pi}{T} \tau\right)\right]^{2}
$$

where $F_{Y P}$ is the predicted lift force in the $C F$ direction. $C_{L}$ is lift force coefficient and $\tau$ is a time delay representing a phase difference. For convenience of description, the phase difference $\varphi$ is expressed as,

$$
\varphi=\frac{2 \pi}{T} \tau \times \frac{180}{\pi}\left(^{\circ}\right)
$$

The Equation (16) can be simplified as,

$$
F_{Y P}=\frac{1}{2} \rho C_{L} D L U_{m}^{2}[\cos (\omega t+\varphi)]^{2}, \omega=\frac{2 \pi}{T}
$$

Expanding Equation (18) out, we obtain

$$
F_{Y P}=\frac{1}{4} \rho C_{L} D L U_{m}^{2}+\frac{1}{4} \rho C_{L} D L U_{m}^{2} \cos (2 \omega t+2 \varphi)
$$

From Equation (19), the predicted force can satisfy the main features observed in Section 4.1 and can be divided into two parts-the mean lift force $F_{Y P M}$ and twice the forced motion frequency fluctuating lift force $F_{Y P_{0}}$.

$$
F_{Y P}=F_{Y P M}+F_{Y P^{\circ}}
$$


Through Equation (20), the lift coefficient can be calculated by,

$$
C_{L}=F_{Y M} /\left(1 / 4 \rho D L U_{m}^{2}\right)
$$

where $F_{Y M}$ is the measured mean lift force in the CF direction.

Here, Equation (18) only has two parameters that remain to be determined, the lift coefficient $C_{L}$ and the phase difference $\varphi$. However, if we attempt to directly describe the lift force as the mean and fluctuating component, we need to determine four parameters including the mean value, fluctuating frequency, fluctuating amplitude and phase difference. This novel empirical formula in Equation (18) directly assigns the fluctuating frequency as twice of the frequency of oscillatory flow and the fluctuating amplitude the same as the mean value, which reduced undetermined parameters from four to two. This reduction is directly based on our observation of the main feature of the measured lift force; for example, red line in Figure $6 \mathrm{~b}$. In the following, we demonstrate that such a reduction of parameters still well predicts the fluctuating lift force in a range of $\mathrm{KC}$ number, Froude number and Stokes number.

To determine the value of $\varphi$, the sum of the squared difference $J_{1}(\varphi)$ between the predicted and measured fluctuating force, which can be expressed as,

$$
J_{1}(\varphi)=\sum\left[\left(F_{Y}-F_{Y M}\right)-\left(F_{Y P}-F_{Y P M}\right)\right]^{2}, \quad 0 \leq \varphi \leq 180^{\circ}
$$

Figure 8 presents the value of $J_{1}(\varphi)$ versus phase difference angle $\varphi$ under $\mathrm{KC}=18.8$ at $\beta=11,364$. It can be seen that $J_{1}(\varphi)$ minimizes at $\varphi=21^{\circ}$. Thus, the phase difference angle at this case is adopted as $21^{\circ}$. Using the same method, the value of $\varphi$ for each test case can be obtained. To validate the predicted method, the identified lift coefficient and phase angle are substituted into the predicted formula of Equation (18). The recalculated lift force and measured force in an oscillatory flow are shown in Figure 9 . The blue dashed and red solid line respectively represents the measured and calculated force. The calculated forces are in a good agreement with the measured forces. Therefore, it is feasible and promising to use the prediction formula described in Equation (18) to calculate the hydrodynamic force in the CF direction in an oscillatory flow. Such a good agreement using this novel empirical formula in Equation (18) also provides a potential explanation of the mechanism of the fluctuating lift force with frequency twice of the oscillatory flow, which is worthwhile for further study through flow visualization and CFD.

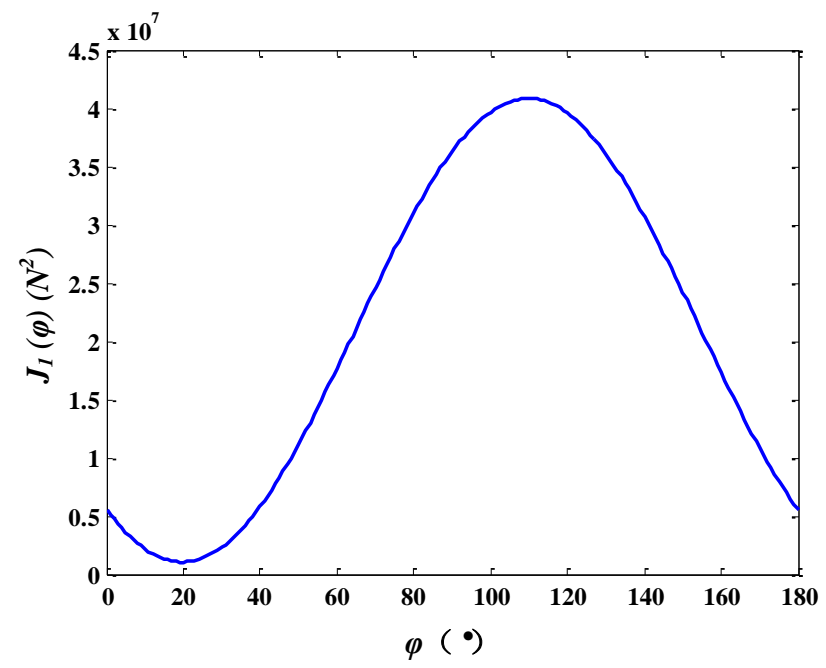

Figure 8. The sum of the squared difference $J_{1}(\varphi)$ between the predicted and measured fluctuating force versus phase difference under $\mathrm{KC}=18.8$ at $\beta=11,364(\mathrm{~A}=0.75 \mathrm{~m}, \mathrm{~T}=5.5 \mathrm{~s})$. 


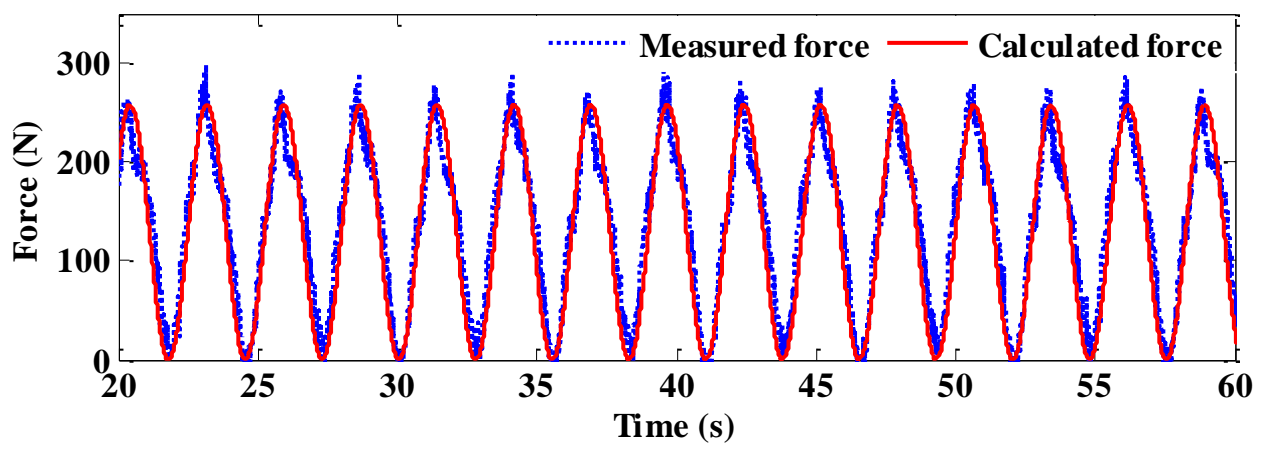

Figure 9. Time history of the measured and calculated hydrodynamic forces for semi-submerged cylinder in the cross-flow (CF) direction under $\mathrm{KC}=18.8$ at $\beta=11,364(\mathrm{~A}=0.75 \mathrm{~m}, \mathrm{~T}=5.5 \mathrm{~s})$.

Moreover, for computational fluid dynamics, this problem is a typical two-phase flow problem, which is not simple to solve. However, this empirical formula can greatly avoid the complicated and time-consuming hydrodynamic calculation problem and will be very efficient and convenient for engineer to calculate the force on semi-submerged cylinder, such as the floating collar of fish farming. However, in real engineering project, the semi-submerged cylinder will not have a constant vertical displacement, which is likely to result in a different lift force. This work mainly focused on the static semi-submerged status of the cylinder, which will also provide a preliminary estimation of the lift force and basic references for further investigation. More systematic experiments will be carried out to determine the influence of vertical and horizontal vibration and even the submerged depths on hydrodynamic load in near future.

\subsection{Drag and Added Mass Coefficients for Semi-Submerged Cylinder}

Combining measured hydrodynamic force and forced motion in the IL direction, we can calculate the drag coefficient $\left(C_{D}\right)$ and added mass coefficient $\left(C_{m}\right)$ using the method proposed in Section 3. To verify the identified method of hydrodynamic coefficients, the hydrodynamic force is recalculated through Equation (8) by identified drag and added mass coefficient as shown in Figure 10. The blue dashed line and red solid line represent the measured force and calculated force, respectively. The good consistency is witnessed between measured and calculated value. This demonstrates the validity of the identification method for hydrodynamics of semi-submerged cylinder under an oscillatory flow.

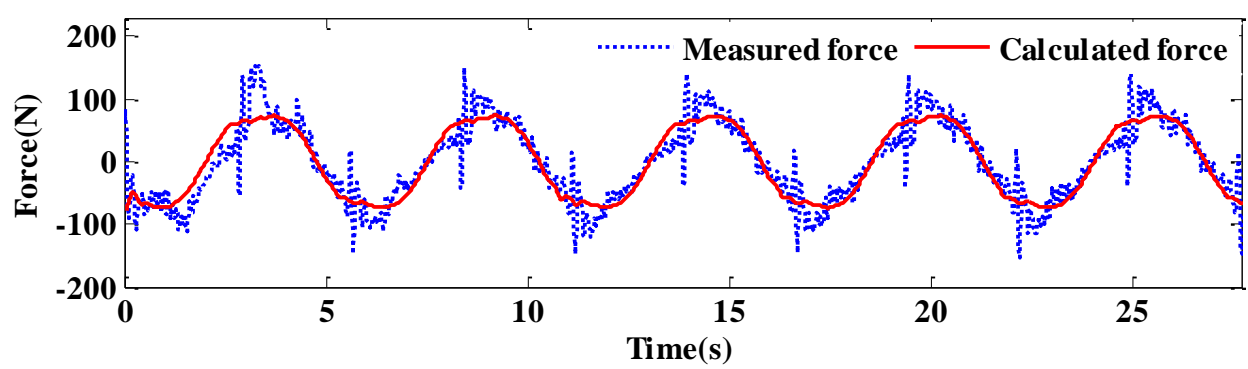

Figure 10. Time history of the measured and calculated hydrodynamic forces for semi-submerged cylinder in the in-line (IL) direction under $\mathrm{KC}=18.8$ at $\beta=11,364(\mathrm{~A}=0.75 \mathrm{~m}, \mathrm{~T}=5.5 \mathrm{~s})$.

Figure 11 shows the drag and added mass coefficients distribution with $\mathrm{KC}$ number under different Stokes number $\beta$. The relationship between $C_{D}$ and $\beta$ is indistinct as shown in Figure 11a. When the $\mathrm{KC}$ number is less than 20, the discrete distribution features is very obvious. Therefore, the Stokes number would more greatly affect the drag coefficient at this condition $(K C<20)$ but does not show a significant influence on drag coefficient under $K C>20$. As the value of $\beta$ increase, the drag coefficient increases under smaller $\mathrm{KC}$ number $(\mathrm{KC}<20)$ but decreases under larger $\mathrm{KC}$ number $(K C>20)$. Different from the results of drag coefficients, the distinctive features is found that $C_{m}$ 
increase with $\mathrm{KC}$ and $\beta$ number as shown in Figure 11b. For the semi-submerged cylinder, the free surface effects cannot be ignored. Thus, the Froude number will be an important parameter to describe the hydrodynamic coefficients $[14,19]$. Further, Figure 12 present the drag and added mass coefficient changing with Froude number under the same Stokes number. The drag coefficient in Figure 12a shows the disorderly and irregular characteristics. The relationship between $C_{D}$ and Fr cannot be directly concluded under the same Stokes number. As for the added mass coefficient of semi-submerged cylinder, the positive correlation between $C_{m}$ versus $\mathrm{Fr}$ is also obviously seen under the same $\beta$ value as shown in Figure 12b. Under the same KC number, it is clear that hydrodynamic coefficients are significantly related to the Froude number as illustrated in Figure 13 . When $\mathrm{KC}<20$, the drag coefficient decreases as Froude number increases. An unexpected phenomenon is observed that the drag coefficient increase linearly with Froude number as KC $>20$ as shown in Figure 13a. With the increase of KC number, the relationship of drag coefficient has a significant evolution trend with Froude number, nonlinear decay changing to linear increase. Additionally, the added mass coefficients show a nonlinear $(K C<20)$ and a linear $(K C>20)$ increase trend similar to the trend of drag coefficient under KC $>20$. The evolution trend from nonlinear to linear uptrend of the added mass coefficient can also be witnessed. From above observation, it can be inferred that using the Froude number and KC number to predict the hydrodynamic coefficient of semi-submerged cylinder in an oscillatory flow is a promising way in engineering project, especially for larger KC number $(K C>20)$.
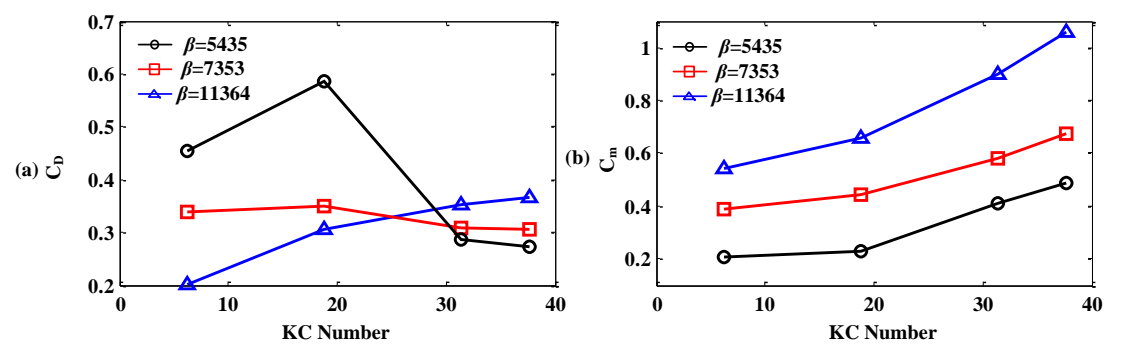

Figure 11. Drag and added mass coefficients changing with KC number under different Stokes number ((a) drag coefficients; (b) added mass coefficient).
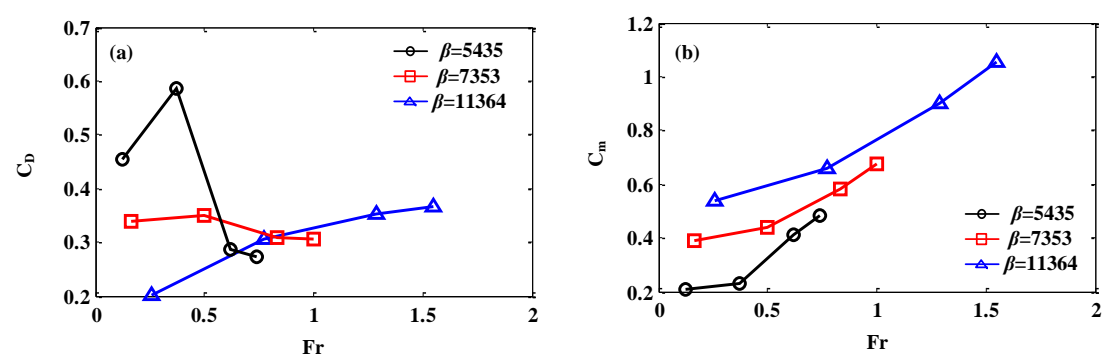

Figure 12. Drag and added mass coefficients changing with Froude number under different Stokes number ((a) drag coefficients; (b) added mass coefficient).
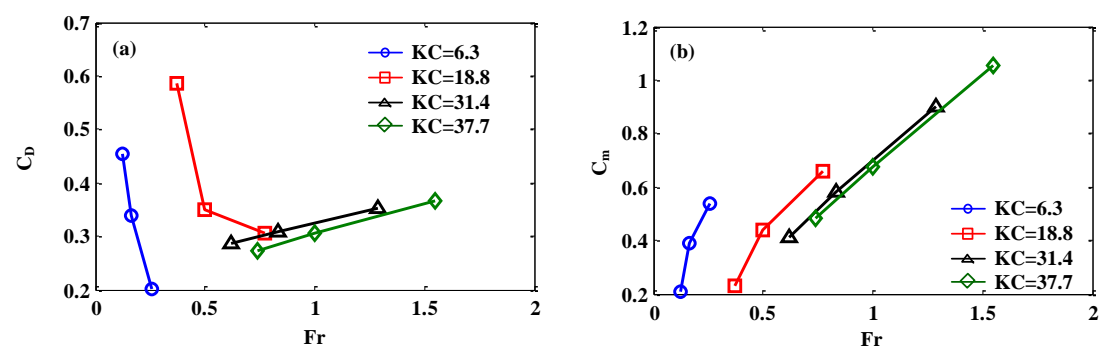

Figure 13. Drag and added mass coefficients changing with Froude number under different KC number ((a) drag coefficients; (b) added mass coefficient). 


\subsection{Lift Coefficients for Semi-Submerged Cylinder}

Through the above identified method described in Section 4.2, the lift coefficients and phase angle are determined and summarized in Figures 14-16. Figure 14 shows the lift coefficients and phase angle changing with KC number under the same Stokes number. The maximum lift coefficient can reach 1.5, which is even larger than the typical value of drag coefficient for fully submerged cylinder $\left(C_{D}=1.2\right)$ as shown in Figure 14a. Therefore, such large lift force for semi-submerged cylinder cannot be ignored in the related structure design and engineering projects.
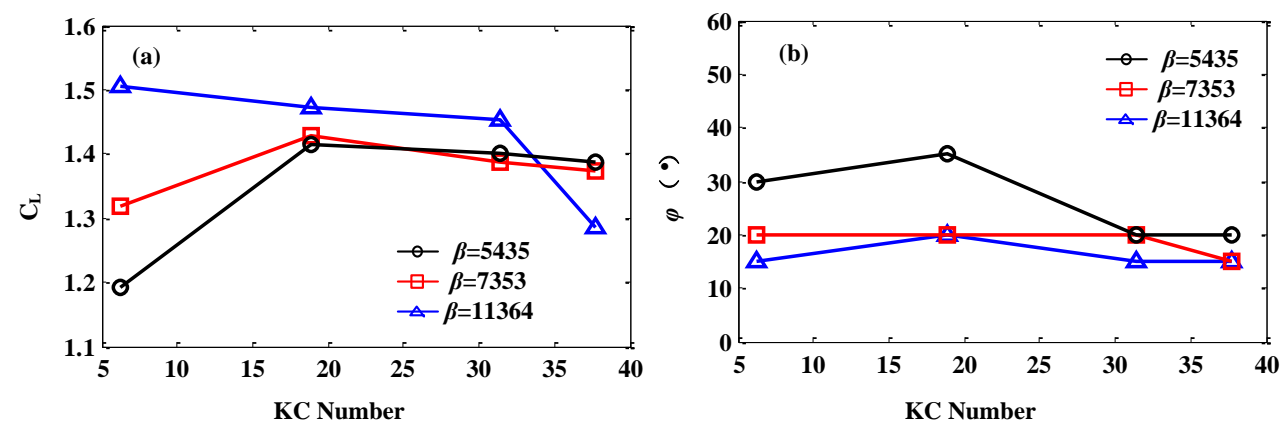

Figure 14. Lift coefficient and phase difference angle changing with KC number under different Stokes number ((a) lift coefficients; (b) phase difference angle).
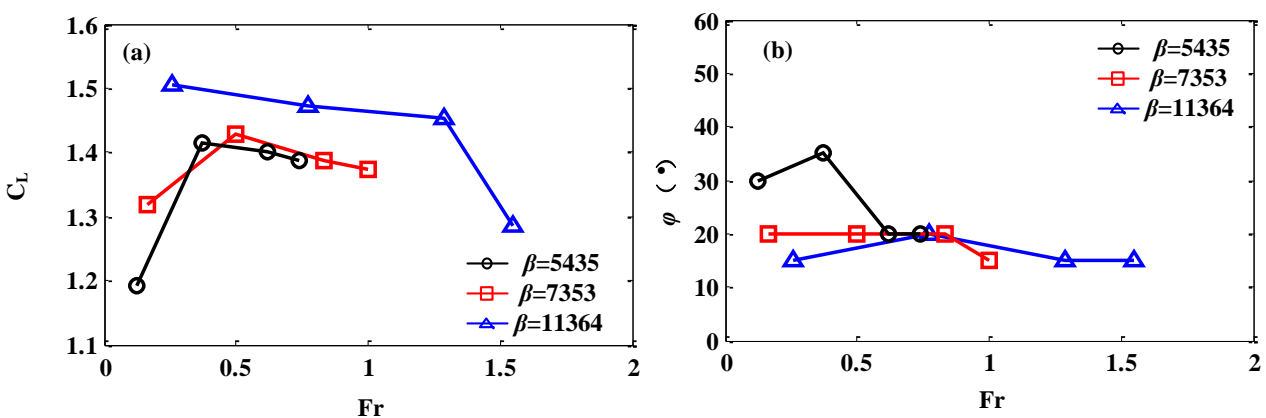

Figure 15. Lift coefficient and phase difference angle changing with Froude number under different KC number ((a) lift coefficients; (b) phase difference angle).
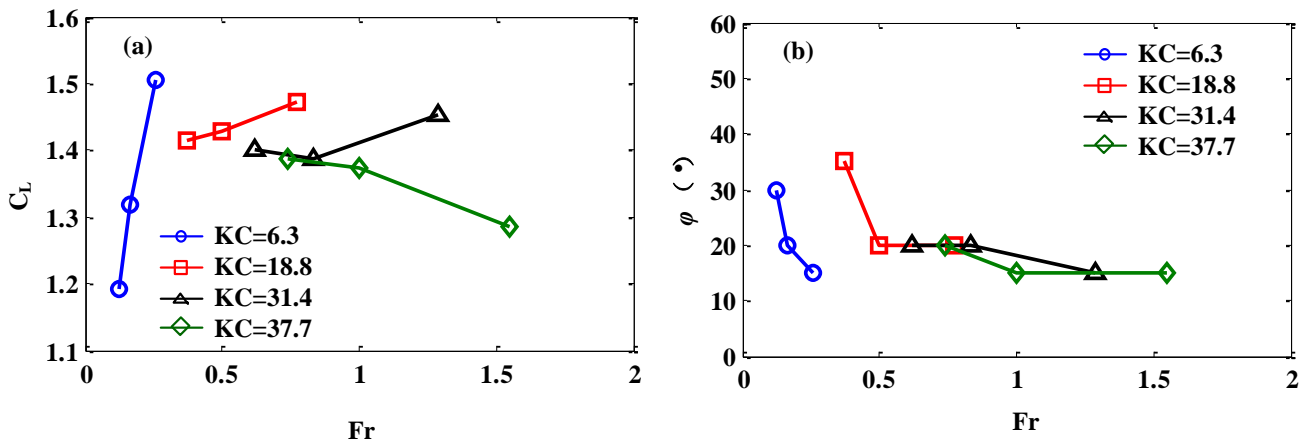

Figure 16. Lift coefficient and phase difference angle changing with Froude number under different KC number ((a) lift coefficients; (b) phase difference angle).

Similar to the drag coefficient, the discrete distribution can be also observed in lift coefficients when $\mathrm{KC}<15$. The lift coefficient varies from 1.19 to 1.51 . At this region, the lift coefficients are significantly affected by the Stokes number and seem to increase with the value of the parameter $\beta$. When $\mathrm{KC}>15$ and $\beta<11,364$, the lift coefficients are stable at approximately 1.40 with $\mathrm{KC}$ number. The effects of Stokes number and KC number seem minor. Under higher Stokes number $(\beta=11,364)$, an unexpected drop of $C_{L}$ occurs. $C_{L}$ decreases from 1.45 to 1.25 . This phenomenon will be discussed 
in next section. As for phase difference angle, the angles are stable with $\mathrm{KC}$ number under the same Stokes number. $\varphi$ stabilizes at approximately $16^{\circ}, 19^{\circ}$ and $26^{\circ}$ for $\beta=11,364,7353$ and 5435 , respectively. Different from lift coefficient, the angle decreases with Stokes number. Slight fluctuation can be found in the angle distribution under smaller Stokes number $(\beta=5435)$.

To further investigate the effects of Froude number, Figure 15 illustrates the above two values versus Froude number under the same Stokes number. The stable trend of $C_{L}$ can be seen in the region of Fr ranging from 0.3 to 1.3 as presented in Figure 15a. Relatively dispersed distribution is also found under lower Froude number. Sharp downward trend happens when Fr is around 1.5. In addition, there is slightly obvious phenomenon that the lift coefficient rises with Stokes number when the Froude number is greater than 0.5. Figure $15 \mathrm{~b}$ shows the phase angle with Froude number under the same Stokes number. More discrete features of the phase distribution can be observed under $\mathrm{Fr}<0.5$, while stable and slight downtrend appears under $\mathrm{Fr}>0.5$.

Under the same $K C$ number, the distribution of $C_{L}$ versus Froude number is presented in Figure 16. $C_{L}$ increases with Froude number when $K C \leq 31.4$, while decreases with $F r$ for $\mathrm{KC}>31.4$. Similar evolutionary phenomenon with drag and added mass coefficient can also be found in lift coefficient. However, there is no linear relationship between $C_{L}$ and $F r$. The trend of the $C_{L}$ changes from rapid rise to flat increase and finally shows a downward trend. As for the phase angle, $\varphi$ decreases with Froude number, especially under smaller KC number $(K C \leq 18.8)$ shown in Figure 16b. Rapid descend of the phase angle changes to gradual fall with $\mathrm{KC}$ number increasing. According to above comparison and analysis, it is more reasonable to use the Froude number and $\mathrm{KC}$ number to predict the lift coefficient and phase angle when the Stokes number and Froude number are in the conflict for the semi-submerged cylinder.

\subsection{Effects of Instantaneous Overtopping}

Besides the slamming phenomenon, the overtopping is another tough problem for semi-submerged cylinder in an oscillatory flow $[18,19,24]$. In Section 4.3, an unexpected drop of the lift coefficient was observed in Figures 14 and 15. To further investigate the hydrodynamic mechanism behind this phenomenon, the water surface waveforms, the corresponding water surface sketches at the maximum forced motion velocity, the time histories of forced motion and hydrodynamic force in both CF and IL directions are compared and shown in Figure 17. Under the same Stokes number, the upstream water level of the cylinder keeps rising with KC number increasing as shown in Figure 17a,e,i,m. To clearly illustrate the water level change, the corresponding sketches are shown in Figure $17 \mathrm{~b}, \mathrm{f}, \mathrm{g}, \mathrm{n}$. The symbol " $\mathrm{H}$ " represents the upstream water level. When $\mathrm{KC}=37.70$, the upstream water was over the top of the semi-submerged cylinder, which is termed as "overtopping" phenomenon $[18,19]$. Different from that in steady flow, the overtopping only happens at around the time of forced motion velocity amplitude. We redefine the phenomenon as "instantaneous overtopping." Combining with the lift coefficient distribution, we observe that the drop phenomenon happens as the instantaneous overtopping occurs. The reason can be attributed to the changes of the flow field asymmetry. When the overtopping occurs, this asymmetry weakens, which results in a reduction in the lift coefficient. The same phenomenon was also found in the results under the steady flow reported by Ren et al. (2019) [19]. However, the effects of overtopping in an oscillatory flow were not seen in drag and added mass coefficients. Moreover, the drag force and lift force appear relatively high frequency fluctuation in the peak area as shown in red rectangle part of Figure $17 \mathrm{c}, \mathrm{d}, \mathrm{g}, \mathrm{h}, \mathrm{k}, \mathrm{l}, \mathrm{o}, \mathrm{p}$. The fluctuation of the hydrodynamic force is also attributed to the instantaneous "overtopping" phenomenon at forced motion velocity amplitude. The extreme surface distortions and water fragmentation caused by the instantaneous "overtopping" phenomenon result in this higher frequency hydrodynamic force. Limited by the quantity of experimental data, more systematic experiments will be conducted to study these effects of instantaneous "overtopping" phenomenon. 
$\mathrm{KC}=6.28 \quad \mathrm{~A}=0.25 \mathrm{~m} \quad \mathrm{~T}=5.5 \mathrm{~s} \quad \beta=11364$
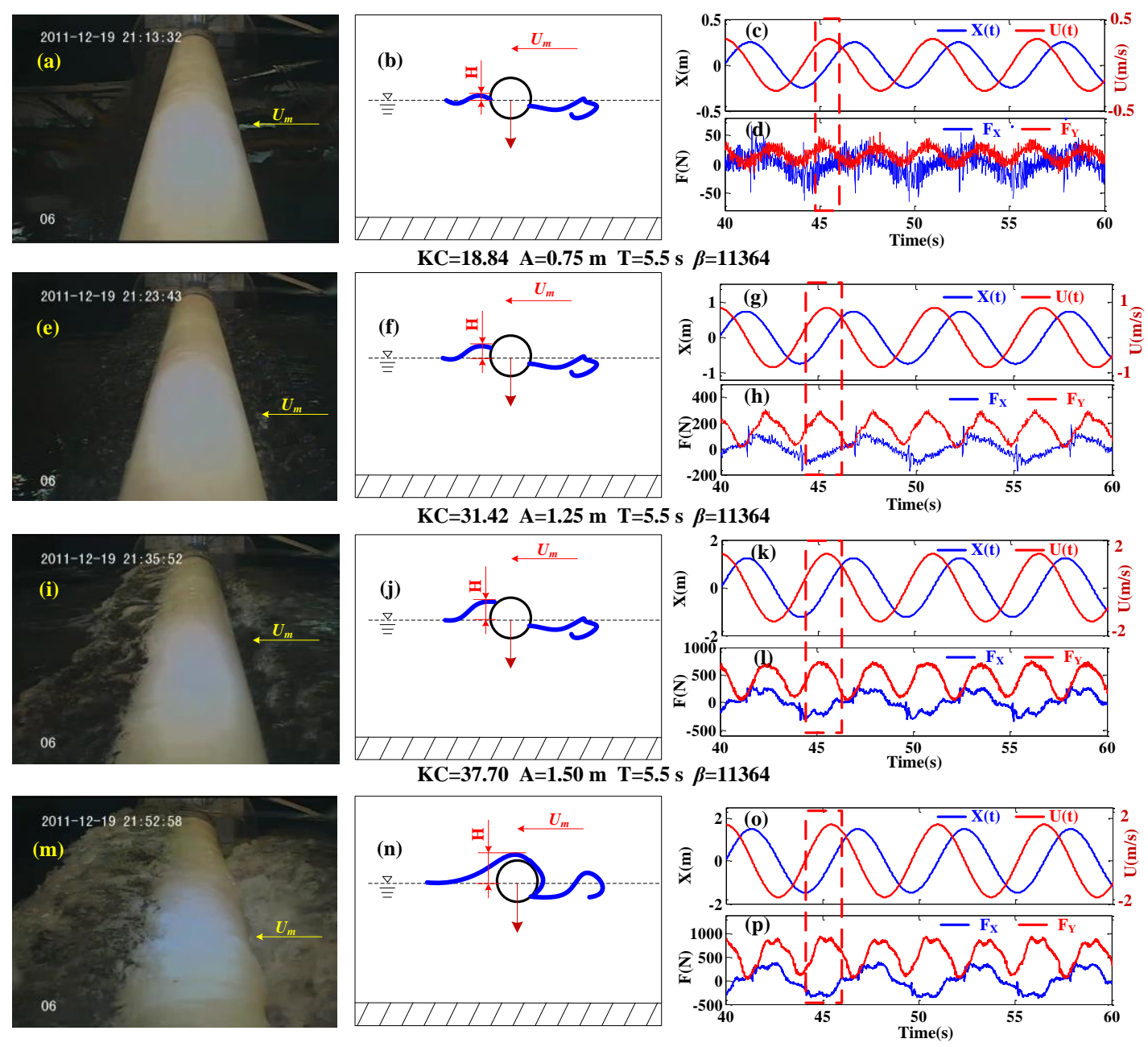

Figure 17. The water surface waveforms and the sketches at the maximum forced motion velocity, the time histories of forced motion and hydrodynamic force in both CF and IL directions changing with KC number under the same Stokes number. $(\mathbf{a}, \mathbf{e}, \mathbf{i}, \mathbf{m})$ The pictures of water surface waveforms at the maximum forced motion velocity; $(\mathbf{b}, \mathbf{f}, \mathbf{j}, \mathbf{n})$ Corresponding sketches of water surface waveforms; $(\mathbf{c}, \mathbf{d}, \mathbf{g}, \mathbf{h}, \mathbf{k}, \mathbf{l}, \mathbf{o}, \mathbf{p})$ time histories of forced motion and hydrodynamic forces.

\section{Conclusions}

In this paper, the hydrodynamic loads on semi-submerged cylinder in an oscillatory flow are experimentally studied. To generate the equivalent oscillatory flow, the semi-submerged cylinder is forced to oscillate in various combinations of different amplitudes and periods. The KC number, Stokes number and Froude number respectively ranges from 6.3 to 37.7 , from 5434 to 11,364 and from 0.12 to 1.55. The hydrodynamic loads in both IL and CF directions are measured. The main hydrodynamic features are observed and summarized. The empirical prediction formula of the lift force in the $\mathrm{CF}$ direction and the relationships among the hydrodynamic coefficient, $\mathrm{KC}$ number, Stokes number and Froude number are further investigated. The main conclusions are as follows:

(1) Similar to the results in steady flow, a significant mean downward lift force of semi-submerged cylinder can also be witnessed. The dominant frequencies of fluctuating lift forces are always twice the forced oscillation frequency.

(2) Based on the observed load features, a novel empirical prediction formula of the lift force on semi-submerged cylinder under oscillatory flow was first proposed, which expresses the lift forces as proportional to the square of the oscillatory flow velocity in the IL direction. This novel empirical 
formula directly explains the underlying mechanism of twice frequency oscillation observed in lift forces, which also provides a more accurate prediction of dynamic load. This formula can well describe the main lift force features including mean value, oscillation that with frequency twice of the oscillatory flow frequency and the leading phase difference.

(3) The maximum lift coefficient can be found to be 1.5, which is larger than the typical values of drag coefficient $\left(C_{D}=1.2\right)$ for a fully submerged cylinder with infinite depth. Such a large lift force may cause related structure damage and should be considered in real engineering projects.

(4) With KC number increasing, the significant evolution trends between drag coefficients, added mass coefficients, lift coefficient and phase difference angle with Froude number under the same $\mathrm{KC}$ number were found. It suggests that using the Froude number and $\mathrm{KC}$ number to predict the hydrodynamic coefficient is more reasonable when the Stokes number and Froude number are in the conflict for the semi-submerged cylinder.

Author Contributions: Conceptualization, H.R., S.F. and C.L.; methodology, H.R.; validation, M.Z., Y.X., S.D. and S.F.; formal analysis, H.R.; investigation, H.R., S.F.; resources, S.F.; data curation, H.R.; writing-original draft preparation, H.R.; writing - review and editing, H.R.; visualization, H.R.; supervision, S.F.; project administration, S.F.; funding acquisition, S.F. All authors have read and agreed to the published version of the manuscript.

Funding: This research was funded by the National Natural Science Foundation of China (No. 51825903, 51909159), the Shanghai Science and Technology Program (No. 19XD1402000, 19JC1412800, 19JC1412801), the Joint Funds of the National Natural Science Foundation of China (No. U19B2013), the Key Projects for Intergovernmental Cooperation in International Science (No. 2018YFE0125100).

Acknowledgments: The authors gratefully acknowledge the support from the National Natural Science Foundation of China (No. 51825903, 51909159), the Shanghai Science and Technology Program (No. 19XD1402000, 19JC1412800, 19JC1412801), the Joint Funds of the National Natural Science Foundation of China (No. U19B2013), the Key Projects for Intergovernmental Cooperation in International Science (No. 2018YFE0125100).

Conflicts of Interest: The authors declare no conflict of interest.

\section{References}

1. Morison, J.R.; Johnson, J.W.; O’Brien, M.P. Experimental studies of forces on piles. Coast. Eng. Proc. 1953, 1, 25. [CrossRef]

2. Morison, J.R.; Johnson, J.W.; Schaaf, S.A. The Force Exerted by Surface Waves on Piles. J. Pet. Technol. 1950, 2, 149-154. [CrossRef]

3. Keulegan, G.H. Forces on cylinders and plates in an oscillating fluid. J. Res. Natl. Bur. Stand. Res. Pap. 1958, 2857, 423-440. [CrossRef]

4. Mercier, J.A. Large Amplitude Oscillations of a Circular Cylinder in Low Speed Stream. Ph.D. Thesis, Stevens Institute of Technology, Hoboken, NJ, USA, 1973.

5. Justesen, P. Hydrodynamic Forces on Large Cylinders in Oscillatory Flow. J. Waterw. Port Coast. Ocean Eng. 1989, 115, 497-514. [CrossRef]

6. Sarpkaya, T. Force on a circular cylinder in viscous oscillatory flow at low Keulegan-Carpenter numbers. J. Fluid Mech. 2006, 165, 61-71. [CrossRef]

7. Sarpkaya, T. Vortex Shedding and Resistance in Harmonic Flow about Smooth and Rough Circular Cylinders at High Reynolds Numbers; Interim Report; Naval Postgraduate School: Monterey, CA, USA, 1976.

8. Williamson, C.H.K. Sinusoidal flow relative to circular cylinders. J. Fluid Mech. 1985, 155, 141-174. [CrossRef]

9. Tatsuno, M.; Bearman, P.W. A visual study of the flow around an oscillating circular cylinder at low Keulegan-Carpenter numbers and low Stokes numbers. J. Fluid Mech. 1990, 211, 157. [CrossRef]

10. Roshko, A. Flow Forces on a Cylinder Near a Wall or Near another Cylinder. In Proceedings of the 2nd US Nation Conference on Wind Engineering Research, Fort Collins, CO, USA, 1-7 January 1975.

11. Bearman, P.W.; Zdravkovich, M.M. Flow around a circular cylinder near a plane boundary. J. Fluid Mech. 1978, 89, 33-47. [CrossRef]

12. Lei, C.; Cheng, L.; Kavanagh, K. Re-examination of the effect of a plane boundary on force and vortex shedding of a circular cylinder. J. Wind Eng. Ind. Aerodyn. 1999, 80, 263-286. [CrossRef]

13. Reichl, P.; Hourigan, K.; Thompson, M.C. Flow past a cylinder close to a free surface. J. Fluid Mech. 1997, 533, 269-296. [CrossRef] 
14. Sheridan, J.; Lin, J.C.; Rockwell, D. Metastable states of a cylinder wake adjacent to a free surface. Phys. Fluids 1995, 7, 2099-2101. [CrossRef]

15. Triantafyllou, G.S.; Dimas, A.A. Interaction of two-dimensional separated flows with a free surface at low Froude numbers. Phys. Fluids 1989, 1, 1813-1821. [CrossRef]

16. Kristiansen, D.; Faltinsen, O.M. A study of wave loads on fixed horizontal cylinders in the free surface. In Proceedings of the 8th International Conference on Hydrodynamics, Nates, France, 30 September-3 October 2008.

17. Kristiansen, D. Wave Induced Effects on Floaters of Aquaculture Plants; IMT: Trondheim, Norway, 2010.

18. Fu, S.; Xu, Y.; Hu, K.; Zhang, Y. Experimental investigation on hydrodynamics of floating cylinder in oscillatory and steady flows by forced oscillation test. Mar. Struct. 2013, 34, 41-55. [CrossRef]

19. Ren, H.; Xu, Y.; Zhang, M.; Deng, S.; Sun, H. Hydrodynamic forces on a partially submerged cylinder at high Reynolds number in a steady flow. Appl. Ocean Res. 2019, 88, 160-169. [CrossRef]

20. Achenbach, E.; Heinecke, E. On Vortex Shedding from Smooth and Rough Cylinders in the Range of Reynolds Number Multiplied by $10^{3}$ to Multiplied by $10^{6}$. J. Fluid Mech. 1981, 109, 239-251. [CrossRef]

21. Chakrabarti, S.K. Hydrodynamics of Offshore Structures; Wit Press: Southampton, UK, 1987.

22. Sumer, B.M.; Fredsøe, J. Hydrodynamics Around Cylindrical Structures; World Scientific: Singapore, 1997.

23. Bozkaya, C.; Kocabiyik, S. Free surface wave interaction with an oscillating cylinder. Appl. Math. Lett. 2014, 27, 79-84. [CrossRef]

24. Hu, K.; Fu, S.; Ma, L.; Song, L. Nonlinear Hydrodynamics of a Floating Cylinder in Oscillatory Flow Alone and Combined with a Current. J. Waterw. Port Coast. Ocean Eng. 2016, 143, 04016015. [CrossRef]

(C) 2020 by the authors. Licensee MDPI, Basel, Switzerland. This article is an open access article distributed under the terms and conditions of the Creative Commons Attribution (CC BY) license (http://creativecommons.org/licenses/by/4.0/). 